\title{
EL CÁNCER DE PRÓSTATA EN LA COMUNIDAD DE MADRID EN EL AÑO 2000. III - ESTUDIO DE EXTENSIÓN TUMORAL(*)
}

\author{
F. HERRANZ AMO ${ }^{1}$, F. ARIAS FUNEZ ${ }^{2}$, M. ARRIZABALAGA MORENO ${ }^{3}$, \\ F.J. CALAHORRA FERNÁNDEZ ${ }^{4}$, J. CARBALLIDO RODRÍGUEZ ${ }^{5}$, R. DIZ RODRÍGUEZ ${ }^{6}$, \\ J.A. HERRERO PAYO ${ }^{7}$, C. LLORENTE ABARCA ${ }^{8}$, J.C. MARTÍN MARTÍNEZ ${ }^{9}$, \\ L. MARTÍNEZ-PIÑEIRO LORENZO ${ }^{10}$, R. MÍNGUEZ MARTÍNEZ ${ }^{11}$, J. MORENO SIERRA ${ }^{12}$, \\ A. RODRÍGUEZ ANTOLÍN ${ }^{13}$, J.C. TAMAYO RUİZ ${ }^{14}$, J. TURO ANTONA ${ }^{15}$ \\ ${ }^{1}$ H.G.U. Gregorio Marañón. ${ }^{2}$ H. Ramón y Cajal. ${ }^{3}$ H. de Móstoles. ${ }^{4}$ Fundación Jiménez Díaz. ${ }^{5}$ H. Puerta de \\ Hierro. ${ }^{6} \mathrm{H}$. del Aire. ${ }^{7} \mathrm{H}$. Severo Ochoa. ${ }^{8} \mathrm{H}$. Fundación de Alcorcón. ${ }^{9} \mathrm{H}$. El Escorial. ${ }^{10} \mathrm{H}$. de La Paz. ${ }^{11} \mathrm{H}$. de La \\ Princesa. ${ }^{12}$ H. Clínico. ${ }^{13}$ H. 12 de Octubre. ${ }^{14} \mathrm{H}$. Príncipe de Asturias $y{ }^{15} \mathrm{H}$. Militar Gómez Ulla. Madrid.
}

Actas Urol Esp. 27 (6): 411-417, 2003

\section{RESUMEN}

EL CÁNCER DE PRÓSTATA EN LA COMUNIDAD DE MADRID EN EL AÑO 2000. III.- ESTUDIO DE EXTENSIÓN TUMORAL(*)

OBJETIVO: Conocer si existe relación entre las variables de riesgo de metástasis y la utilización de las pruebas de imagen en el estudio de extensión de los pacientes con cáncer de próstata diagnosticados en la Comunidad de Madrid en el año 2000.

MATERIAL Y MÉTODO: Se analizaron 1.127 pacientes en los que se conocían los datos sobre el estudio de extensión tumoral. Se relacionó la realización o no de gammagrafía ósea y tomografía computarizada con las variables de riesgo de presentar metástasis descritas en la literatura (PSA, Gleason y estadio) y con el tratamiento aplicado.

RESULTADOS: El porcentaje de pacientes con variables de riesgo de metástasis en los que se realizó gammagrafia ósea fue superior entre un $7 \%$ y $14 \%$ que los que no las presentaban. No existió diferencias en la realización de tomografía computarizada en función de las variables de riesgo. En los pacientes tratados con radioterapia se realizaron más pruebas de imagen en igualdad de variables de riesgo que en los tratados mediante prostatectomía.

CONCLUSIÓN: Según las recomendaciones de la literatura se utilizaron un excesivo número de pruebas de imagen en el estudio de extensión en los pacientes sin variables de riesgo de metástasis. Sería conveniente la realización de un estudio a nivel de nuestra Comunidad para comprobar si con nuestra prevalencia de enfermedad metastásica es posible aplicar dichas recomendaciones.

PALABRAS CLAVE: Cáncer de próstata. Estadio. Estudio de extensión.

\section{ABSTRACT}

PROSTATE CANCER IN THE AUTONOMOUS COMMUNITY OF MADRID IN 2000. III.- TUMOUR EXTENSION STUDY

OBJECTIVE: To identify a potential relationship between two variables, risk of metastasis and use of imaging techniques, in an extension study in prostate cancer patients diagnosed in the Autonomous Community of Madrid in 2000.

MATERIAL AND METHODS: 1,127 patients with available data on the tumour extension study were analysed. Performance and non performance of bone scans and CTs were correlated to risk variables for developing metastasis as described in the literature (PSA, Gleason and stage) and to therapy administered.

RESULTS: The proportion of patients with risk variables for metastasis when bone scans were performed was between $7 \%$ to $14 \%$ greater than in patients with no variables. No differences were seen for CTs based on risk variables. With matching risk variables, more imaging techniques were used in patients receiving radiotherapy that in those managed with prostatectomy.

CONCLUSION: Based on current recommendations imaging techniques were used in excess in the extension study in patients with no risk variables for metastasis. Conduct of a further study in the Autonomous Community seems advisable to confirm the likelihood of implementing such recommendations considering our prevalence of metastatic disease.

KEY WORDS: Prostate cancer. Stage. Extension study.

${ }^{*}$ ) Este artículo forma parte del estudio "El cáncer de próstata en la Comunidad de Madrid en el año 2000" auspiciado por la Sociedad Urológica Madrileña y financiado por AstraZéneca. 
$\mathrm{E}_{\mathrm{c}}^{1}$ estudio de extensión tumoral clásico del cáncer de próstata (CP) se basa en los hallazgos al tacto rectal, la tomografía computarizada (TC) y la gammagrafía ósea (GO). Debido a la migración de estadio (aumento de diagnósticos en estadios precoces), la baja sensibilidad de la $\mathrm{TC}^{1}$ y la baja especificidad de la $\mathrm{GO}^{2}$ se recomienda que las pruebas de imagen del estudio de extensión se realicen en función variables de riesgo de metástasis como son: el PSA, tacto rectal y score de Gleason. Varios autores ${ }^{1-3}$ y organizaciones $^{4,5}$ han formulado una serie de recomendaciones para la realización de pruebas de imagen en el estudio de extensión del $\mathrm{CP}$ en base a la relación coste-eficacia, aunque no existe un consenso internacionalmente aceptado.

En nuestro país los autores ${ }^{6-8}$ que han tratado este tema recomendaban la realización de la gammagrafía ósea de forma sistemática como parte del estudio de extensión tumoral. Pero hay que tener en cuenta que son series históricas en las que la prevalencia de enfermedad metastásica oscilaba entre el $21 \%^{8}$ y el $57,6 \%{ }^{6}$. Esta recomendación era lógica ya que la elevada prevalencia de enfermedad metastásica influía negativamente en el valor predictivo negativo ${ }^{9}$ de la prueba, que oscilaba entre el $87,1 \%^{6}$ y el $91,5 \%{ }^{8}$. Pero la prevalencia de enfermedad metastásica en el estudio:"El cáncer de próstata en la Comunidad de Madrid en el año 2000", del que están extraídos los datos de este artículo, fue del 12,5\% que es similar a la que presentaba la serie de Chybowski $^{2}(13,6 \%)$ cuando recomendó la no realización de $\mathrm{GO}$ en los pacientes con cáncer de próstata y PSA menor de $20 \mathrm{ng} / \mathrm{ml}$.

En este artículo analizamos como se realizó el estudio de extensión tumoral en los pacientes de cáncer de próstata diagnosticados en la Comunidad de Madrid en el año 2000 y su relación con las variables de riesgo de existencia de metástasis.

\section{MATERIAL Y MÉTODO}

El ámbito del estudio y la metodología es la misma que la descrita en el artículo: "El cáncer de próstata en la Comunidad de Madrid en el año 2000. I - Incidencia".

El objetivo del artículo es conocer la relación existente entre las pruebas de imagen (TC y GO) realizadas en el estudio de extensión tumoral y las variables de riesgo de afectación ganglionar o metástasis óseas.

Las variables de riesgo de metástasis óseas utilizadas en este estudio se han recogido de la literatura existente ${ }^{1-5}$. Para la realización de la GO son: PSA mayor o menor de $10 \mathrm{ng} / \mathrm{ml}$., score de Gleason mayor o menor de 8, tacto rectal menor o igual a T2 o mayor que T2 y la combinación de tacto rectal y PSA. Para la realización de TC son las mismas variables pero el punto de corte del PSA se establece en $20 \mathrm{ng} / \mathrm{ml}$.

El estudio se realizó sobre los 1.745 pacientes remitidos por los Hospitales participantes en el estudio. En 1.127 pacientes existían datos sobre la realización o no del estudio de extensión (en 1.036 casos se realizó al menos una prueba de extensión tumoral y en 91 no se creyó oportuno realizar ninguna prueba antes de indicar el tratamiento) y en 618 casos se desconocía si se había realizado o no estudio de extensión. Todos los pacientes del Área 2 fueron estudiados mediante GO y TC, por lo tanto estos casos sólo computaron para el estudio general de la Comunidad y no para la estratificación por Áreas. Las Áreas 5 y 10 no enviaron datos sobre el estudio de extensión.

\section{RESULTADOS}

De los 1.127 pacientes con datos conocidos sobre el estudio de extensión, a 804 (71\%) se realizó GO, a 765 (68\%) TC, GO y TC se realizó a 687 (61\%) pacientes y GO o TC a 882 (78\%).

La edad media de los pacientes a los que se les

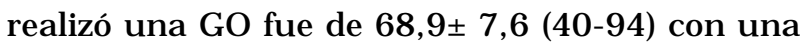
mediana de 69 años, frente a $71 \pm 7,5$ (53-89) con una mediana de 71 años de los pacientes en los que no se realizó. La edad media para los pacientes a los que se les realizó una TC fue de 68,5 \pm 7,3 (40-94) con una media de 69 años, frente a $71,9 \pm 8$ (52-91) con una mediana de 72 años de los pacientes en los que no se realizó.

En los pacientes con patología asociada se realizó GO en el 79,4\% y TC en el $72,8 \%$, frente al $76,8 \%$ y al $74,5 \%$ respectivamente en los pacientes sin patología asociada.

El porcentaje de pacientes con variables de riesgo de metástasis óseas en las que se realizó GO fue más elevado que los que no las presentaban, pero la diferencia fue pequeña, entre el $7 \%$ 
y el 14\% (Tabla I). En los pacientes con variables de riesgo de afectación ganglionar la diferencia entre realizar o no un TC no existía o se invertía, entre el $0 \%$ y el $-2 \%$ (Tabla II). Los resultados al estratificar por Áreas Sanitarias se detallan en las Tablas III y IV.
Según el tratamiento recibido observamos que el porcentaje de pacientes con GO y TC en los pacientes tratados mediante radioterapia es superior a los pacientes tratados mediante prostatectomía. En los pacientes tratados mediante manipulación hormonal el porcentaje de TC era

\section{TABLA I}

RELACIÓN ENTRE LAS VARIABLES DE RIESGO PARA TENER METÁSTASIS ÓSEAS Y LA REALIZACIÓN DE GAMMAGRAFÍA ÓSEA (GO) EN EL ESTUDIO DE EXTENSIÓN TUMORAL

\begin{tabular}{|l|c|c|c|}
\hline Variable & Pacientes & Go - si (\%) & Go - no (\%) \\
\hline PSA $>10$ & 589 & $449(76 \%)$ & $140(24 \%)$ \\
\hline PSA $\leq 10$ & 524 & $350(67 \%)$ & $174(33 \%)$ \\
\hline Gleason $\geq 8$ & 214 & $177(83 \%)$ & $37(17 \%)$ \\
\hline Gleason < & 904 & $621(69 \%)$ & $283(31 \%)$ \\
\hline Estadio $\geq$ T3 & 181 & $143(79 \%)$ & $38(21 \%)$ \\
\hline Estadio $<$ T3 & 944 & $661(70 \%)$ & $283(30 \%)$ \\
\hline Estadio $\leq$ T2 + PSA > 10 & 421 & $313(74 \%)$ & $108(26 \%)$ \\
\hline Estadio $\leq$ T2 + PSA $\leq 10$ & 514 & $344(67 \%)$ & $170(33 \%)$ \\
\hline
\end{tabular}

TABLA II

RELACIÓN ENTRE LAS VARIABLES DE RIESGO PARA TENER METÁSTASIS GANGLIONARES Y LA REALIZACIÓN DE TOMOGRAFÍA COMPUTERIZADA (TC) EN EL ESTUDIO DE EXTENSIÓN TUMORAL

\begin{tabular}{||l|c|c|c|}
\hline \hline Variable & Pacientes & TC - si (\%) & TC - no (\%) \\
\hline PSA > 20 & 316 & $210(67 \%)$ & $106(34 \%)$ \\
\hline PSA $\leq 20$ & 797 & $548(69 \%)$ & $249(31 \%)$ \\
\hline Gleason $\geq 8$ & 214 & $146(68 \%)$ & $68(32 \%)$ \\
\hline Gleason < & 904 & $615(68 \%)$ & $289(32 \%)$ \\
\hline Estadio $\geq$ T3 & 181 & $122(67 \%)$ & $59(33 \%)$ \\
\hline Estadio < T3 & 944 & $643(68 \%)$ & $301(32 \%)$ \\
\hline Estadio $>$ T2 + PSA $>20$ & 148 & $101(68 \%)$ & $47(32 \%)$ \\
\hline Estadio $\leq$ T2 + PSA $\leq 20$ & 768 & $529(69 \%)$ & $239(31 \%)$ \\
\hline
\end{tabular}

\section{TABLA III}

RELACIÓN ENTRE LAS VARIABLES DE RIESGO PARA TENER METÁSTASIS ÓSEAS Y LA REALIZACIÓN DE GAMMAGRAFÍA ÓSEA (EN PORCENTAJES) EN EL ESTUDIO DE EXTENSIÓN TUMORAL SEGÚN LAS DISTINTAS ÁREAS SANITARIAS DE LA COMUNIDAD DE MADRID

\begin{tabular}{||l|c|c|c|c|c|c|c|c|c|}
\hline Variable & A1 & A3 & A4 & A6 & A7 & A8 & A9 & A12 & HM \\
\hline PSA $>10$ & 87 & 77 & 86 & 91 & 43 & 64 & 89 & 36 & 86 \\
\hline PSA $\leq 10$ & 88 & 79 & 85 & 97 & 21 & 14 & 47 & 33 & 100 \\
\hline Gleason $\geq 8$ & 91 & 80 & 92 & 92 & 58 & 56 & 80 & 50 & 82 \\
\hline Gleason < 8 & 86 & 75 & 84 & 93 & 27 & 27 & 66 & 34 & 93 \\
\hline Estadio $\geq$ T3 & 97 & 86 & 84 & 75 & 68 & 56 & 89 & 25 & 71 \\
\hline Estadio < T3 & 86 & 75 & 86 & 95 & 29 & 28 & 60 & 36 & 94 \\
\hline Estadio $\leq$ T2 + PSA > 10 & 86 & 71 & 87 & 94 & 34 & 69 & 83 & 40 & 88 \\
\hline Estadio $\leq$ T2 + PSA $\leq 10$ & 88 & 79 & 85 & 97 & 19 & 12 & 49 & 33 & 100 \\
\hline
\end{tabular}




\section{TABLA IV}

RELACIÓN ENTRE LAS VARIABLES DE RIESGO PARA TENER METÁSTASIS ÓSEAS Y LA REALIZACIÓN DE GAMMAGRAFÍA ÓSEA (EN PORCENTAJES) EN EL ESTUDIO DE EXTENSIÓN TUMORAL SEGÚN LAS DISTINTAS ÁREAS SANITARIAS DE LA COMUNIDAD DE MADRID

\begin{tabular}{||l|c|c|c|c|c|c|c|c|c||}
\hline Variable & A1 & A3 & A4 & A6 & A7 & A8 & A9 & A12 & HM \\
\hline PSA $>20$ & 72 & 53 & 56 & 100 & 41 & 40 & 86 & 65 & 75 \\
\hline PSA $\leq 20$ & 87 & 37 & 80 & 90 & 27 & 34 & 62 & 74 & 96 \\
\hline Gleason $\geq 8$ & 79 & 70 & 57 & 100 & 33 & 41 & 80 & $0\left(^{*}\right)$ & 73 \\
\hline Gleason < 8 & 84 & 39 & 81 & 92 & 33 & 33 & 67 & 74 & 100 \\
\hline Estadio $\geq$ T3 & 81 & 50 & 56 & 88 & 40 & 48 & 83 & 50 & 86 \\
\hline Estadio < T3 & 83 & 41 & 78 & 93 & 31 & 32 & 64 & 75 & 94 \\
\hline Estadio > T2 + PSA > 20 & 85 & 58 & 56 & 100 & 38 & 38 & 86 & 50 & 50 \\
\hline Estadio $\leq$ T2 + PSA $\leq 20$ & 88 & 38 & 78 & 92 & 26 & 31 & 63 & 74 & 96 \\
\hline
\end{tabular}

$(*)$ Sólo dos pacientes.

muy inferior a los sujetos tratados con radioterapia o prostatectomía (Tabla V). Al estratificar según el tratamiento aplicado y las variables de riesgo de metástasis (Tabla VI) observamos que existe una tendencia a realizar más GO y TC en los pacientes sometidos a radioterapia que en los que se trataron con prostatectomía.

En la Tabla VII se detalla el porcentaje de pruebas de imagen en los pacientes con factores pronósticos de altísimo riesgo de metástasis o con escasa expectativa de vida. En los pacientes con estudio de extensión realizado y PSA $>100$ $\mathrm{ng} / \mathrm{ml}$., el $68 \%$ presentaban metástasis óseas.

\section{DISCUSIÓN}

Desde que Chybowski y cols. en 1991 recomendaran la no realización de GO en los pacientes con cáncer de próstata y valores de PSA inferiores a $20 \mathrm{ng} / \mathrm{ml}$ debido a su baja rentabilidad (VPN del $99,7 \%$ ), varios autores ${ }^{1,3,10-12}$ han confirmado la baja rentabilidad de esta prueba recomendando diferentes puntos de cortes del PSA, generalmente $\leq 10 \mathrm{ng} / \mathrm{ml} \mathrm{o} \leq 20 \mathrm{ng} / \mathrm{ml}$. Glave y cols ${ }^{13}$ incluyen como variables de riesgo de metástasis óseas, ade-

\section{TABLA V}

RELACIÓN ENTRE LA REALIZACIÓN DE GAMMAGRAFÍA ÓSEA (GO) Y TOMOGRAFÍA COMPUTERIZADA (TC) EN EL ESTUDIO DE EXTENSIÓN Y EL TRATAMIENTO RECIBIDO

\begin{tabular}{||l|c|c||}
\hline \hline Tratamiento & GO - si & TC - si \\
\hline Prostatectomía & $262(71 \%)$ & $276(74 \%)$ \\
\hline Radioterapia & $245(87 \%)$ & $244(87 \%)$ \\
\hline Manipulación hormonal & $270(70 \%)$ & $220(57 \%)$ \\
\hline
\end{tabular}

\section{TABLA VI}

REALIZACIÓN DE GAMMAGRAFÍA ÓSEA (GO) Y TOMOGRAFÍA COMPUTARIZADA (TC) SEGÚN EL TRATAMIENTO RECIBIDO Y LAS VARIABLES DE RIESGO DE METÁSTASIS

\begin{tabular}{||l|c|c|}
\hline Variable & Prostatectomía & Radioterapia \\
\hline PSA $\leq \mathbf{1 0}$ ng/ ml & & \\
- GO - si & $63 \%$ & $83 \%$ \\
- TC - si & $72,5 \%$ & $85 \%$ \\
\hline PSA > 10 ng/ ml & & \\
- GO - si & $82 \%$ & $90,5 \%$ \\
- TC - si & $78 \%$ & $88 \%$ \\
\hline PSA $\leq \mathbf{2 0}$ ng/ ml & & \\
- GO - si & $69,5 \%$ & $85 \%$ \\
- TC - si & $74 \%$ & $85 \%$ \\
\hline PSA > 20 ng/ ml & & \\
- GO - si & $91 \%$ & $94 \%$ \\
- TC - si & $86 \%$ & $94 \%$ \\
\hline Gleason < & & $87 \%$ \\
- GO - si & $70 \%$ & $85,5 \%$ \\
- TC - si & $75 \%$ & $86 \%$ \\
\hline Gleason $\geq \mathbf{8}$ & & $85 \%$ \\
- GO - si & $71 \%$ & \\
- TC - si & $75 \%$ & $89 \%$ \\
\hline Estadio T1-2 & & \\
- GO - si & $71 \%$ & \\
- TC - si & & \\
\hline Estadio T3-4 & & \\
- GO - si & & \\
- TC - si & & \\
\hline
\end{tabular}

(*) Menos de 5 pacientes. 


\section{TABLA VII}

REALIZACIÓN DE GAMMAGRAFÍA ÓSEA (GO) Y TOMOGRAFÍA COMPUTARIZADA (TC) EN FUNCIÓN DE VARIABLES DE MUY ALTO RIESGO DE METÁSTASIS O CUANDO LA EXPECTATIVA DE VIDA ES MUY ESCASA

\begin{tabular}{||l|c|c|c||}
\hline Variable & Pacientes & Go - si & TC - si \\
\hline PSA > 100 ng/ml & 100 & $82 \%$ & $58 \%$ \\
\hline Edad $\geq 75$ años & 303 & $65 \%$ & $53,5 \%$ \\
\hline Edad $\geq 80$ años & 98 & $56 \%$ & $41 \%$ \\
\hline PSA > 100 y Edad $\geq 75$ & 36 & $81 \%$ & $50 \%$ \\
\hline PSA > 100 y Edad $\geq 80$ & 13 & $77 \%$ & $46 \%$ \\
\hline
\end{tabular}

más de un PSA > $10 \mathrm{ng} / \mathrm{ml}$., un estadio localmente avanzado (T3) o un grado pobremente diferenciado (Gleason $\geq 8$ ) en la biopsia. En otros trabajos $^{6-8,14}$, entre las que se encuentran todas las series nacionales publicadas, recomiendan la realización de GO a todos los pacientes independientemente de las variables pronosticas. Esto es debido a que la elevada prevalencia de enfermedad metastásica existente influye negativamente en el valor predictivo negativo de la prueba.

O`Dowd y cols. realizaron una revisión de la literatura en el periodo 1975-1996 seleccionando 142 artículos válidos y llegaron a las siguientes conclusiones: 1) la GO sólo es necesaria en los pacientes asintomáticos con un PSA $>10 \mathrm{ng} / \mathrm{ml}$; 2) sólo se debe de realizar TC o RM a los pacientes con PSA $>20 \mathrm{ng} / \mathrm{ml}$. o Gleason $\geq 8$ o tumor localmente avanzado al tacto rectal (T3 o T4).

Varias organizaciones han emitido sus recomendaciones en cuanto al estudio de extensión del cáncer de próstata en sus guías de práctica clínica. The National Comprehesive Cancer Network (NCCN) recomendaba en $1996^{4}$ la realización de: 1 . GO en los pacientes con tumor T1-T2 con un PSA $>10 \mathrm{ng} / \mathrm{ml}$. o Gleason $\geq 8$ o tumor T3-T4 o en los pacientes sintomáticos; y 2. TC o RM en los pacientes con tumor T3-T4. En su versión del año $2000^{16}$ se mantienen los mismos criterios para el estudio de extensión, especificando que sus recomendaciones son de nivel 2A (estas recomendaciones se han tomado por consenso uniforme entre los miembros del NCCN, basadas en un bajo nivel de evidencia incluyendo la experiencia clínica). El RCR (Real College of Radiologists) ${ }^{5}$ recomienda la omisión de GO en los pacientes con un tumor bien diferenciado y unos niveles de PSA inferiores a $20 \mathrm{ng} / \mathrm{ml}$., con un grado B de recomendación (avalados por estudios clínicos bien diseñados pero no aleatorizados). La EAU (European Association of Urology) ${ }^{17}$ recomendó en sus guías de práctica clínica del año 2001 que: 1) no está indicada la GO en los pacientes con PSA < 10 $\mathrm{ng} / \mathrm{ml}$. y tumor bien o moderadamente diferenciado; y 2) la TC o RM sólo estaría indicada en los pacientes en los que se plantea un tratamiento curativo y en los sujetos con alto riesgo de afectación ganglionar (los criterios que maneja son: PSA $>20 \mathrm{ng} / \mathrm{ml}$., estadio T2b o superior, tumor pobremente diferenciado e invasión tumoral perineural).

Como podemos comprobar aunque existe una tendencia en la literatura mundial a no realizar pruebas de imagen en el estudio de extensión en los pacientes con bajo riesgo de metástasis en función de variables como PSA, estadio y grado de diferenciación o Gleason, no existe un acuerdo en que variables utilizar.

En nuestro estudio los Urólogos de la Comunidad de Madrid no tienen en cuenta las variables pronosticas de metástasis óseas y de afectación ganglionar al realizar el estudio de extensión. Las diferencias al realizar una GO entre los pacientes con y sin variables de riesgo de metástasis óseas oscila entre el $7 \%$ y el $14 \%$ y entre el $0 \%$ y el $-2 \%$ en la realización de un TC en función de las variables de riesgo de afectación ganglionar. En la estratificación por Áreas Sanitarias observamos que algunas Áreas tienen una tendencia mayor que otras a seguir las recomendaciones de la literatura.

En 1997 Plawker y cols. ${ }^{18}$ realizaron una encuesta sobre el manejo del cáncer de próstata a 1.500 Urólogos de USA elegidos aleatoriamente en la base de datos de la AUA (American Urological Association) teniendo un $43 \%$ de respuestas válidas. El 52,4\% de los encuestados respondió que realizaba GO independientemente de los niveles de PSA, el 29,5\% sólo cuando PSA > $10 \mathrm{ng} / \mathrm{ml}$. y el $11,9 \%$ sólo cuando PSA $>20 \mathrm{ng} / \mathrm{ml}$. El 28,6\% realizaba TC independientemente de los niveles de PSA, el $12,6 \%$ si el PSA > $10 \mathrm{ng} / \mathrm{ml}$. y el $12,4 \%$ cuando el PSA $>20 \mathrm{ng} / \mathrm{ml}$., llegando a la conclusión de que existía un uso excesivo de ambas pruebas de imagen. Fleshner y cols. ${ }^{19}$ realizaron una encuesta sobre el manejo del cáncer de próstata a 700 Urólogos norteamericanos y 350 canadienses 
elegidos aleatoriamente en las bases de datos de ambas sociedades profesionales. El porcentaje de cuestionarios contestados válidos fue del 45,3\% en los norteamericanos y del $79,2 \%$ en los canadienses. Los Urólogos norteamericanos realizaron TC y GO de forma rutinaria en el 23,3\% y el $40 \%$, nunca en el 33,8\% y el 3,8\% y de forma selectiva en el 41,8\% y el 51,9\% respectivamente, mientras que los Urólogos canadienses lo realizaron de manera rutinaria en el $12 \%$ y $33,1 \%$, nunca en el $60,9 \%$ y $6,8 \%$ y de forma selectiva en el $21,9 \%$ y $59 \%$ respectivamente.

Kindrick y cols. ${ }^{20}$ basándose en la base de datos CaPSURE (Cancer of the Prostate Strategic Urologic Research Endeavor) en el periodo comprendido entre 1989 y 1997 estudiaron si se seguían en la clínica las recomendaciones de la literatura con respecto al uso de pruebas de imagen en el estudio de extensión en los pacientes con cáncer de próstata. Se recogieron 3.557 casos de 29 Urólogos, comprobando que no hubo cambios a lo largo del tiempo y que había una utilización excesiva de pruebas de imagen en pacientes de bajo riesgo de tener enfermedad metastásica, de forma muy similar a lo obtenido en nuestro estudio.

Albertsen y cols. ${ }^{21}$ determinan el rendimiento de las pruebas de imagen (TC y GO) en el estudio de extensión en 3.690 pacientes diagnosticados de cáncer de próstata entre 1994 y 1995 extraídos del SEER (Surveillance, Epidemiology and End Results Program). Confirman el bajo rendimiento de dichas pruebas, la variabilidad geográfica existente (diferente práctica regional e impacto de los seguros sanitarios) y el mayor uso de TC y GO en los pacientes tratados con radioterapia. Recomiendan realizar TC y GO sólo a los pacientes con PSA > $20 \mathrm{ng} / \mathrm{ml}$. o Gleason 8-10, ya que en los pacientes que no cumplen estos criterios el riesgo de metástasis oscila entre el $0 \%$ y el $5 \%$.

Saidal y cols. ${ }^{22}$ extraen una muestra aleatoria que representa el 5\% de los beneficiarios de todos los Medicare entre los años 1991-1996. Encontrando entre ellos 17.586 pacientes diagnosticados de cáncer de próstata localizado $(67,5 \%$ tratados mediante radioterapia y el $32,5 \%$ restante con prostatectomía), observan que en los pacientes tratados con radioterapia se realizaron más GO y TC de forma estadísticamente significativa que en los tratados con prostatectomía y que existía una importante variabilidad geográfica. En nuestro estudio a igualdad de variables de riesgo de metástasis también existe una tendencia a realizar más pruebas de imagen en los pacientes que fueron tratados con radioterapia, probablemente para poder cumplir los requisitos impuestos por los Servicios de Radioterapia.

Recientemente se ha publicado un estudio ${ }^{23}$ basado en la base de datos CaPSURE sobre 4.966 pacientes para comparar la realización de pruebas de imagen en el estudio de extensión antes de 1997 y después de dicho año. Los pacientes se dividen en 3 grupos de riesgo: 1) bajo: PSA $<10$ ng/ml., Gleason $<7$ y estadio clínico T1 o T2a; 2) intermedio: PSA entre 10,1 y $15 \mathrm{ng} / \mathrm{ml}$., Gleason de 7 o estadio clínico T2b; 3) alto: PSA $>15 \mathrm{ng} / \mathrm{ml}$., Gleason $>7$ o estadio clínico T3 o T4. En el estudio se observa una importante reducción en la realización de pruebas de imagen del 63\%, 25,9\% y del $11,4 \%$ en los 3 grupos de riesgo, sobre todo en el último año del estudio. A pesar de esta reducción tan importante los autores encuentran que en la cuarta parte de los pacientes del grupo de bajo riesgo y en más de la mitad de los de riesgo intermedio se siguen realizando pruebas de imagen.

Si el objetivo de no realizar estudios de imagen en los pacientes con escasa probabilidad de tener enfermedad metastásica es aumentar la relación coste-beneficio, de la misma forma no se deberían de realizar en los pacientes en los que debido a la elevada probabilidad de metástasis o escasa expectativa de vida el estudio de extensión no modificaría la elección del tratamiento. Rana y cols. $^{24}$ encontraron que todos los pacientes con PSA $>100 \mathrm{ng} / \mathrm{ml}$. tenían metástasis óseas, aunque hay que tener en cuenta que su serie era pequeña (60 pacientes). Herranz y cols. ${ }^{25}$ encontraron en su serie de 555 pacientes con cáncer de próstata estudiados mediante GO que el $63 \%$ de los pacientes con PSA $>100 \mathrm{ng} / \mathrm{ml}$, tenían metástasis óseas demostrables. En nuestra serie el $68 \%$ de los sujetos con PSA $>100 \mathrm{ng} / \mathrm{ml}$, presentaban metástasis óseas en la GO. Un PSA $>100$ $\mathrm{ng} / \mathrm{ml}$, una expectativa de vida corta (edad superior a 75, 80 años) o la asociación de ambas no supuso la disminución en la realización de GO, en cambio estas variables disminuyeron de forma moderada la realización de TC entre un $10 \%$ y un $27 \%$ con respecto a la media del estudio. 
Los Urólogos de la Comunidad de Madrid no seguimos las recomendaciones de la literatura referentes a la realización de pruebas de imagen en el estudio de extensión de los pacientes con cáncer de próstata. Realizamos un número excesivo de pruebas de imagen en los pacientes con CP y baja probabilidad de diseminación metastásica. Sería conveniente la realización de un estudio a nivel de nuestra Comunidad para comprobar si con nuestra prevalencia de enfermedad metastásica es posible aplicar dichas recomendaciones.

\section{REFERENCIAS}

1. LEVRAN Z, GONZÁLEZ JA, DIOKNO AC et al.: Are pelvic computed tomography, bone scan and pelvic lymphadenectomy necessary in the staging of prostatic cancer?. Br J Urol 1995; 75: 778-781.

2. CHYBOWSKI FM, KELLER JJL, BERGSTRALH EJ, OESTERLING JE.: Predicting radionuclide boen scans indings in patient with newly diagnosed untreated prostate cancer: prostate specific antigen is superior to all other clinical parameters. J Urol 1991; 145: 313318.

3. O`DOWD GJ, VELTRI RW, OROZCO R, MILLER MC, OESTERLING JE.: Update on the appropiate staging evaluation for newly diagnosed prostate cancer. $J$ Urol 1997; 158: 687-698.

4. BAKER LH, HANKS G, GERSHENSON D et al.: NCCN prostate cancer practice guidelines. The National Comprhensive Cancer Network. Oncology 1996; 10 (suppl): 265-288.

5. The Royal College of Radiologists' Clinical Oncology Information Network British Association of Urological Surgeons. Guidelines on the management of prostate cancer. BJU International 1999; 84: 987-1014.

6. MOROTE J, OLONA M, VILLA J y cols.: ¿Podemos prescindir de la gammagrafia ósea en el estadiaje del cáncer de próstata?. Actas Urol Esp 1992; 16: 487-490.

7. PERTUSA PEÑA C, ALBISU TRISTÁN A, LLARENA IBARGUREN R.: ¿Cuándo realiza rastreo óseo en el cáncer de próstata?. Actas Urol Esp 1994; 18: 387-396.

8. HERRANZ AMO F, DÍEZ CORDERO JM, VERDÚ TARTAJO F y cols.: Correlación entre el antígeno específico prostático, el grado tumoral y el estadio local con la gammagrafía ósea en el estadiaje de los pacientes con cáncer de próstata. Arch Esp Urol 1997; 50: 253-258.

9. CHICHARRO MOLERO J, BURGOS RODRIGUEZ RB.: Test diagnósticos. Pags:159-176. En: Burgos Rodríguez R: Metodología de investigación y escritura científica en clínica. $2^{a}$ ed. Escuela Andaluza de Salud Pública. Granada. 1996.

10. OESTERLING JE, MARTÍN SK, BERGSTRALH EJ, LOWE FC.: The use of prostate-specific antigen in staging patients with newly diagnosed prostate cancer. JAMA 1993; 269: 57-60.

11. HAUKAAS S, ROERVIK J, HALVORSEN OJ, FOELLINGS M.: When is bone scintigraphy necessary in the assessment of newly diagnosed, untreated prostate cancer?. Br J Urol 1997; 79: 770-776.
12. WOLFF JM, ZIMNY M, BORCHES H, WILDBERGER J, BUELL U, JAKSE G.: Is prostate-specific antigen a reliable marker of bone metastasis in patients with newly diagnosed cancer of the prostate?. Eur Urol 1998; 33: 376-381.

13. GLEAVE ME, COUPLAND D, DRACHENBERG D et al.: Ability of serum prostate-specific antigen levels to predict normal bone scans in patients with newly diagnosed prostate cancer. Urology 1996; 47: 708-712.

14. MILLER PD, EARDLEY I, KIRBY RS.: Prostate specific antigen and bone scan correlation in the staging and monitoring of patients with prostate cancer. $\mathrm{Br} J$ Urol 1992; 70: 295-298.

15. O`DOWD GJ, VELTRI RW, OROZCO R, MILLER MC, OESTERLING JE.: Update on the apropiate staging evaluation for newly diagnosed prostate cancer. J Urol 1997; 158: 687-698.

16. BAKER LH, POW-SANG JM, BAHNSON RR et al.: NCCN practice guidelines for prostate cancer. Version 2000. Oncology 2000; 14 (11A): 111-119.

17. AUS G, ABBOU CC, PACIK D et al.: Guidelines on prostate cancer. En: E.A.U: guidelines.

18. PLAWKER MW, FLEISHER JM, VAPNEK EM, MACCHIA RJ.: Current trends in prostate cancer diagnosis and staging among united states urologist. J Urol 1997; 158: 1853-1859.

19. FLESHNER N, RAKOVITCH E, KLOTZ L.: Differences between urologist in the united states and Canada in the approach to prostate cancer. J Urol 2000; 163: 1461-1466.

20. KINDRICK AV, GROSSFELD GD, STIER DM, FLANDERS SC, HENNING JM, CARROLL PR.: Use of imaging test for staging newly diagnosed prostate cancer: trends from the capsure database. J Urol 1998; 160: 2102-2106.

21. ALBERTSEN PC, HANLEY JA, HARLAN LC et al.: The positive yield of imaging studies in the evaluation of men with newly diagnosed prostate cancer: a population based analysis. J Urol 2000; 163: 1138-1143.

22. SAIGAL CS, PASHOS CL, HENING JM, LITWIN MS.: Variaions in use imaging in national sample of men with early-stage prostate cancer. Urology 2002; 59: 400-404.

23. COOPERBERG MR, LUBECK DP, GROSSFELD GD, MEHTA SS, CARROLL PR.: Contemporary trends in imaging test utilization for prostate cancer staging: data from the cancer of the Prostate Strategic Urological Research Endeavor. J Urol 2002; 168: 491-495.

24. RANA A, KARAMANIS K, LUCAS MG, CHISHOLM GD.: Identificación of metastatic disease by $\mathrm{T}$ category, Gleason score and serum PSA level in patients with carcinoma of the prostate. Br J Urol 1992; 69: 277-281.

25. HERRANZ AMO F, VERDÚ TARTAJO F, DÍEZ CORDERO JM y cols.: ¿Se puede prescindir de la gammagrafia ósea en el estadiaje del cáncer de próstata?. Comunicación al LXIV Congreso Nacional de Urología. Zaragoza. 1999.

Dr. F. Herranz Amo

C/ Doctor Esquerdo, 155 $5^{\mathrm{a}}-7^{\mathrm{o}} 3$

28007 Madrid

(Trabajo recibido el 19 noviembre de 2002) 